\title{
Spectroscopic observations of the recurrent nova Cl Aquilae during the 2000 outburst $^{\star}$
}

\author{
T. Iijima
}

\author{
Astronomical Observatory of Padova, Asiago Section, Osservatorio Astrofisico, 36012 Asiago (Vi), Italy \\ e-mail: takashi.iijima@oapd.inaf.it
}

Received 25 August 2011 / Accepted 9 June 2012

\begin{abstract}
High- and low-resolution optical spectra of CI Aql were obtained during the outburst in 2000. Multiple absorption components of H I and Fe II lines were detected at the early decline stage. Their radial velocities were roughly $-2500 \mathrm{~km} \mathrm{~s}^{-1},-2200 \mathrm{~km} \mathrm{~s}^{-1}$, $-1700 \mathrm{~km} \mathrm{~s}^{-1}$, and $-1400 \mathrm{~km} \mathrm{~s}^{-1}$, among which only the last components were likely still accelerating during the early decline stage. Prominent emission lines of [O III] and [N II] appeared about one month after light maximum. The duration of the nebular stage, however, was only one month and a few weeks. The ejected gas shells seem to have started to shrink about 70 days after light maximum. The amount of interstellar extinction is estimated to be $E(B-V)=0.92 \pm 0.15$ from the equivalent widths of the diffuse interstellar absorption bands. The helium abundance in the ejecta is estimated to be $N(\mathrm{He})=0.19 \pm 0.05$ and the mass of the ejecta to be about $2 \times 10^{-6} M_{\odot}$. This object has been classified as a U Sco type recurrent nova, but its spectral evolution during the outburst resembled those of T Pyx type recurrent novae. It is doubtful whether the peak of $m_{\mathrm{V}} \cong 9$ mag on 2000 May 5 was the true light maximum or an earlier brighter peak had been overlooked, because the spectral data suggest that the ejections of gas shells occurred prior to the discovery of the outburst on 2000 April 28.
\end{abstract}

Key words. stars: individual: CI Aql - novae, cataclysmic variables

\section{Introduction}

The first historical recorded outburst of the recurrent nova CI Aql was observed in 1917 (Reinmuth 1925). There was probably another outburst in 1941, which was found in a recent survey of old photographic plates (Schaefer 2001). The historical photometric data of CI Aql and the other recurrent novae are summarised by Schaefer (2010).

The last outburst of CI Aql was discovered on 2000 April 28 independently by Takamizawa and Yamamoto (Takamizawa et al. 2000). Kiss et al. (2001) estimated the magnitude and the epoch of light maximum to have been $m_{\mathrm{V}} \cong 9$ mag at 2000 May 5.0 UT, that is JD 2451669.5. Some slightly different parameters are proposed also, those are $m_{\mathrm{V}}=8.8 \mathrm{mag}$ at JD 2451666 by Burlak \& Esipov (2001) and $m_{\mathrm{V}}=9.2 \mathrm{mag}$ at JD 2451671 by Lynch et al. (2004). The parameters of Kiss et al. (2001) are used in this paper. There are, however, some doubts about the epoch and the magnitude of light maximum, which are discussed in Sect. 7.

CI Aql is known as an eclipsing binary system with an orbital period of 0.62 day (Mennickent \& Honeycutt 1995). Matsumoto et al. (2001) and Lederle \& Kimeswenger (2003) analysed the eclipsing light curve after the outburst in 2000 . Lederle \& Kimeswenger (2003) reported that the orbital period slightly lengthened after the outburst. The theoretical models for the outbursts of this object were proposed by Hachisu \& Kato (2001) and Hachisu et al. (2003).

The spectral evolution in the optical region of this object during the outburst in 2000 was reported in some previous works

* Calibrated high-resolution spectra are available at the CDS via anonymous ftp to cdsarc.u-strasbg.fr (130.79.128.5) or via http://cdsarc.u-strasbg.fr/viz-bin/qcat?]/A+A/544/A26
(Burlak \& Esipov 2001; Kiss et al. 2001; Matsumoto et al. 2001). Lynch et al. (2004) performed a detailed analysis for the nearinfrared spectra obtained in 2000 and 2001. In this paper, lowand high-resolution optical spectra obtained in 2000 May, June, and July are presented, which follow the spectral evolution from the early decline stage to the nebular stage. The properties of this object are studied using the spectral data.

\section{Observations}

The high-resolution spectra were obtained with a Reosc echelle spectrograph mounted on the $182 \mathrm{~cm}$ telescope at the Mount Ekar station of the Astronomical Observatory of Padova. The spectral region from $4350 \AA$ to $6900 \AA$ was covered with the resolution of $\lambda / \Delta \lambda \cong 10000$. The low-resolution spectra were obtained at the Bisei Astronomical Observatory in Okayama Japan ${ }^{1}$. A grating spectrograph Bisei mounted on the Cassegrain focus of the $101 \mathrm{~cm}$ telescope was used. The spectral region from $3800 \AA$ to $8000 \AA$ was covered with the resolution of $\lambda / \Delta \lambda \cong 800$. The high- and low-resolution spectra were reduced using the standard tasks of the NOAO IRAF ${ }^{2}$ package at the Asiago station of the Astronomical Observatory of Padova. The spectrophotometric calibrations were performed using the spectra of the standard star $58 \mathrm{Aql}$ (HR7596) obtained during the same nights of the observations. A log of the observations is given in Table 1.

\footnotetext{
1 The observations at the Bisei Observatory were carried out by Matsumoto et al. (2001), who kindly permitted me to use their spectra. 2 IRAF is distributed by NOAO for Research in Astronomy, Inc. under cooperative agreement with the National Science Foundation.
} 
Table 1. Log of spectroscopic observations of CI Aql.

\begin{tabular}{|c|c|c|c|c|c|c|c|}
\hline Date & $\overline{\mathrm{UT}}$ & JD & Days & exp. & Inst. & Range & Sky \\
\hline 2000 & & & & $\mathrm{~s}$ & & $\mathrm{~nm}$ & \\
\hline May 14 & 01:06 & 1678.55 & 9.1 & 600 & Ech & $435-690$ & cloudy \\
\hline May 15 & $00: 56$ & 1679.54 & 10.0 & 600 & " & " & \\
\hline May 15 & 01:08 & 1679.55 & 10.1 & 1800 & " & $"$ & \\
\hline May 16 & 01:05 & 1680.55 & 11.1 & 600 & " & " & \\
\hline May 16 & 01:17 & 1680.55 & 11.1 & 1800 & " & " & \\
\hline May 20 & $17: 49$ & 1685.24 & 15.7 & 420 & Bisei & $380-800$ & \\
\hline May 20 & $17: 59$ & 1685.25 & 15.7 & 420 & " & " & \\
\hline June 9 & $00: 19$ & 1704.51 & 35.0 & 2400 & Ech & $435-690$ & \\
\hline June 10 & $00: 49$ & 1705.53 & 36.0 & 3600 & " & & \\
\hline July 9 & $15: 25$ & 1735.15 & 65.7 & 720 & Bisei & $380-800$ & cloudy \\
\hline July & $17: 10$ & 1735.22 & 65.7 & 900 & & & \\
\hline
\end{tabular}

Notes. UT: Universal time at start of exposure. JD: Julian date - 2450000 . Days: Number of days from light maximum on 2000 May 5 (JD 2451669.5 ).

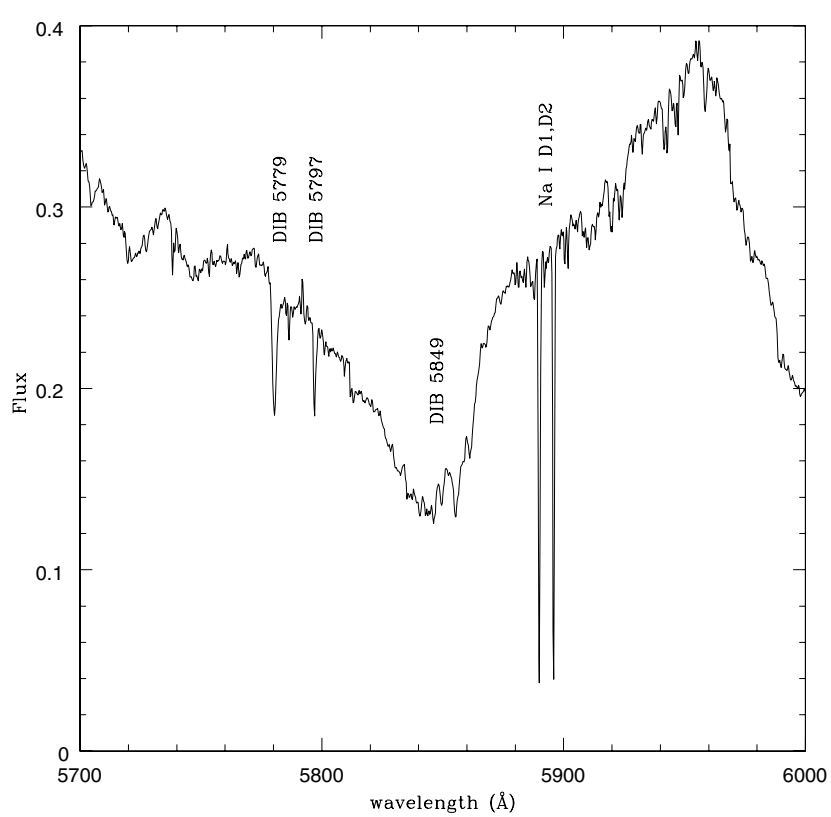

Fig. 1. Diffuse interstellar absorption bands (DIB) and Na I D1 and D2 in the spectrum of CI Aql on 2000 May 15. The ordinate shows the flux in $10^{-12} \mathrm{erg} \mathrm{cm}^{-2} \mathrm{~s}^{-1} \AA^{-1}$.

\section{Interstellar extinction and distance}

\subsection{Interstellar absorption lines and bands}

Rich interstellar absorption lines and bands characterise the optical spectra of this object. Figure 1 shows the spectral region from $5700 \AA$ to $6000 \AA$ of a high-resolution spectrum obtained on 2000 May 15, where the interstellar absorption components of Na I D1 and D2 are deep and the diffuse interstellar absorption bands (DIB) are seen at 5779, 5797, and $5849 \AA$ (Jenniskens \& Désert 1994; Oudmaijer et al. 1997). The equivalent widths of the interstellar absorption components of Na I D1 and D2, and the selected DIB are given in Table 2. These are the means of the values measured on the all available high-resolution spectra. The observational errors are about $\pm 5 \%$.

The interstellar absorption components of Na I D1 and D2 are too deep and probably saturated. Münch (1968) presented the column densities of $\mathrm{Na} \mathrm{I}$ atoms for the ratios of the equivalent widths of $\mathrm{Na}$ I D2/D1 > 1.2 at D1 > 0.65 (Fig. 5 in his article), but we have D2/D1 $=1.1$ at D1 $=0.678$ (Table 2$)$.
Table 2. Equivalent widths of DIB and Na I D1 and D2 and the interstellar extinction.

\begin{tabular}{lccc}
\hline \hline$\lambda$ & $\begin{array}{c}\text { Eq.W. } \\
(\AA)\end{array}$ & Eq.W./E(B-V) & $\begin{array}{c}E(B-V) \\
\text { mag }\end{array}$ \\
\hline 5779 & 0.535 & 0.579 & 0.92 \\
$5797^{\dagger}$ & 0.211 & 0.249 & 0.85 \\
5849 & 0.054 & 0.048 & 1.13 \\
5889 & 0.749 & & \\
5895 & 0.678 & & \\
6203 & 0.098 & 0.107 & 0.92 \\
6270 & 0.093 & 0.076 & 1.22 \\
6613 & 0.255 & 0.231 & 1.10 \\
\hline
\end{tabular}

Notes. ${ }^{(\dagger)}$ Blend of 5795.2 and 5797.1.

Therefore, it is hard to derive the column density of $\mathrm{Na}$ I atoms with the double ratio method of Münch (1968). The amount of interstellar extinction is estimated from the equivalent widths of DIB. The third column of Table 2 gives the ratios of the equivalent widths of DIB to the amount of interstellar extinction given by Jenniskens \& Désert (1994). The interstellar extinctions are presented in the last column. We derive an amount of interstellar extinction $E(B-V)=0.92 \pm 0.15$ as an average of these results.

Kiss et al. (2001) also measured the equivalent widths of DIB, but their spectra contained only two of them at $5849 \AA$ and $6613 \AA$. For this reason, their result has a large error $E(B-V)=$ $0.85 \pm 0.3$. Burlak \& Esipov (2001) derived the amount of interstellar extinction $E(B-V)=0.91 \pm 0.11$ from the Balmer decrement. These results agree with ours within the limits of the errors. On the other hand, Lynch et al. (2004) found a higher value $E(B-V)=1.5 \pm 0.15$ from the ratios of the near-infrared $\mathrm{O}$ I lines. The cause of the disagreement with our result is not known.

\subsection{Distance to $\mathrm{Cl} \mathrm{Aql}$}

It is noteworthy that only one set of interstellar absorption components of Na I D1 and D2 was detected (Fig. 1). Their mean radial velocity is $+13.2 \pm 0.6 \mathrm{~km} \mathrm{~s}^{-1}$ in the frame of the local standard of rest. These results suggest that CI Aql is not reddened by the interstellar matter in the Sagittarius arm. The distance to the near side of the Sagittarius arm in the direction of CI Aql $\left(l=31.7^{\circ}, b=-0.8^{\circ}\right.$ ) is roughly $1 \mathrm{kpc}$ (Kerr \& Westerhout 1965). Therefore, its distance may be less than or does not exceed much $1 \mathrm{kpc}$ at maximum. 


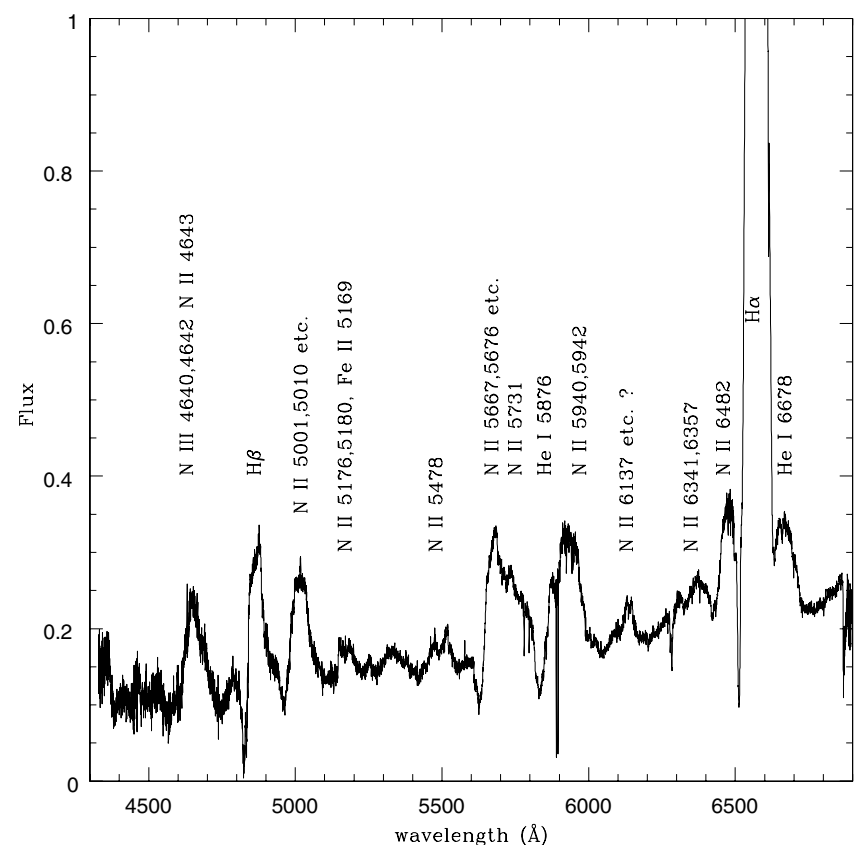

Fig. 2. High-resolution spectrum of CI Aql on 2000 May 14. The ordinate shows the flux in $10^{-12} \mathrm{erg} \mathrm{cm}^{-2} \mathrm{~s}^{-1} \AA^{-1}$.

Absolute $V$ magnitudes of light maxima higher than -7 mag were derived for the outbursts in 1917 and 2000 from the decline rates of luminosity: those are $M_{\mathrm{V}}=-7.35 \pm 0.2$ mag by Kiss et al. (2001), $M_{\mathrm{V}}=-7.4$ mag by Burlak \& Esipov (2001), and $M_{\mathrm{V}}=-7.55 \pm 0.5 \mathrm{mag}$ by Lynch et al. (2004). If we adopt the observed maximum luminosity $m_{\mathrm{V}}=9.0 \mathrm{mag}$ (Kiss et al. 2001) or $m_{\mathrm{V}}=8.8 \mathrm{mag}$ (Burlak \& Esipov 2001) for the outburst in 2000, the above derived absolute maximum luminosities give distances larger than $4.5 \mathrm{kpc}$, adopting $A_{\mathrm{V}}=2.9 \mathrm{mag}$. These distances are inconsistent with the lack of the interstellar absorption components depending on the matter in the Sagittarius arm. It is, however, not sure whether the peak of $m_{\mathrm{V}}=9.0 \mathrm{mag}$ or $8.8 \mathrm{mag}$ was the true light maximum, or if a brighter peak had been overlooked. This problem is discussed in detail in Sect. 7.

A smaller distance $1.55 \mathrm{kpc}$ was derived from a model calculation for the binary system of CI Aql (Hachisu et al. 2003). This distance is consistent with our result.

\section{Spectral evolution}

\subsection{May 14, 15, and 16}

Figure 2 shows a tracing of a high-resolution spectrum obtained on 2000 May 14. All orders of the echelle spectrum are combined using the command scombine of IRAF. The correction for interstellar extinction is not applied to this and all successive tracings of the spectra. The emission lines of H I, He I, N II, and $\mathrm{N}$ III were prominent, while the Fe II emission lines were weak. The emission lines of He I 4922, 5016, and Fe II 4924, and 5018 are prominent in the spectra of usual classical novae. In contrast, the major part of the broad emission band around $5015 \AA$ in this spectrum was likely caused by a blend of N II 5001, 5005, 5010, etc. (Moore 1959) instead of He I 5016 and Fe II 5018. An estimate for the contribution from He I 5015 in the emission band at $5015 \AA$ is given in the next sub-section. The broad absorption line at $4963 \AA$ may have been caused by the absorption components of the N II lines.

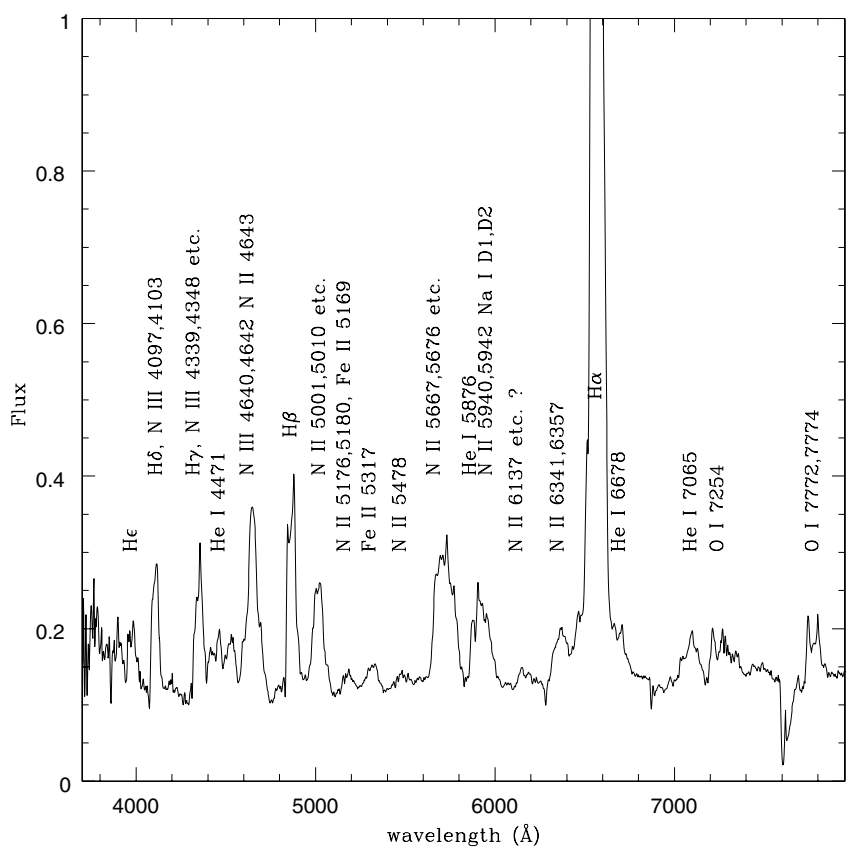

Fig. 3. Low-resolution spectrum of CI Aql on 2000 May 20. The ordinate shows the flux in $10^{-12} \mathrm{erg} \mathrm{cm}^{-2} \mathrm{~s}^{-1} \AA^{-1}$.

The H I emission lines were flanked by P Cygni type absorption components. Detailed arguments for the profiles of the $\mathrm{H}$ I lines are presented in Sect. 5.1. The broad absorption line at $5627 \AA$ was likely caused by N II 5667 and 5680, and that at $5833 \AA$ may have been caused by He I 5876. The high-resolution spectra obtained on 2000 May 15 and 16 displayed nearly the same features. The high intensities of the emission lines of nitrogen ions were also reported in previous works (Burlak \& Esipov 2001; Kiss et al. 2001; Matsumoto et al. 2001).

There was a double peak emission line at $6140 \AA$ (Fig. 2). The wavelengths of the two peaks were $6131 \AA$ and $6147 \AA$. The probable candidates for identification are a blend of N II 36, 6136.9 and 6150.9 and Fe II 46, 6129.7, 6141.0, and 6150.1. However, this is no definitive identification, because the other lines of the same multiplets with higher intensities were not detected, e.g., N II 36, 6167.8 and Fe II 46, 5991.4 (Moore 1959).

\subsection{May 20}

Figure 3 shows a tracing of a low-resolution spectrum obtained on 2000 May 20. The H I emission lines had double peak profiles (see Sect. 5.1). Weak traces of absorption components, blue-shifted by about $-2000 \mathrm{~km} \mathrm{~s}^{-1}$, were seen on $\mathrm{H} \beta$ and the higher members of the Balmer series. The broad emission line at $5015 \AA$ weakened with respect to $\mathrm{H} \beta$, while that at $4640 \AA$, which was mainly caused by the N III lines, strengthened. The emission lines of O I 7254.2 and 7254.5 and O I 7774.2, 7772.0, and 7775.4 (Moore 1959) were prominent in the red region. The intensities of selected emission lines relative to $\mathrm{H} \beta$ were measured and the results are given in Table 3, where $F(\lambda)$ are the measured fluxes and $I(\lambda)$ are the intensities corrected for the interstellar extinction by $E(B-V)=0.92$. The observational errors in the intensities are about $\pm 10 \%$. The values with larger errors are indicated by a colon. The identifications of lines with larger errors in their wavelengths, which are indicated by a colon, are not definitive. 
Table 3. Intensities of selected emission lines relative to $\mathrm{H} \beta=100$ on 2000 May 20.

\begin{tabular}{|c|c|c|c|}
\hline$\lambda_{\mathrm{obs}}$ & $F(\lambda)$ & $I(\lambda)$ & Ident. \\
\hline 3905 & 11 & 24 & H I 3889 \\
\hline 3975 & 23 & 45 & $\mathrm{H} \epsilon$ \\
\hline 4108 & 51 & 92 & H $\delta$, N III 4104, 4097 \\
\hline 4195 & 3 & 6 & N III 4196 \\
\hline 4352 & 56 & 87 & H $\gamma$, N III 4340, 4348 \\
\hline 4420 & 13 & 19 & N II 4427,[Fe II] 4416 \\
\hline 4463 & 12 & 17 & He I 4471,[Fe II] 4452, 4458 \\
\hline 4533 & 21 & 28 & N III 4518, 4524, 4535, etc. \\
\hline 4610: & 20: & 25: & N II 4601, 4607 \\
\hline 4652 & 96 & 114 & N III 4634, 4642, N II 4643 \\
\hline 4700: & 12: & 13: & He II 4686, He I 4713 \\
\hline 4867 & 100 & 100 & $\mathrm{H} \beta$ \\
\hline 5015 & 68 & 61 & N II 5003, 5011, Fe II 5018, He I 5016 \\
\hline 5070: & 5: & 5: & Si II 5056, N II 5045 \\
\hline 5178 & 8 & 6 & N II 5176, 5180, Fe II 5169 \\
\hline 5320 & 14 & 10 & Fe II 5317,5326 \\
\hline 5478 & 6 & 3.7 & N II 5478, 5480, Fe II 5478 \\
\hline 5690: & 79: & 46: & N II 5676, 5680, 5711 \\
\hline 5750: & 73: & 41: & {$[\mathrm{N} \mathrm{II}] 5755$} \\
\hline 5885: & 32: & 17: & He I 5876 \\
\hline 5940: & 53 & 27 & N II 5941, Na I 5890, 5895 \\
\hline 6164: & 9: & 3.9: & N II 6168, 6137, Fe II 6141 etc. \\
\hline 6360: & 17: & 6.9: & Si II 6347, 6371, N II 6357 \\
\hline 6470: & 9: & 3.5: & N II 6482 \\
\hline 6571 & 1110 & 415 & $\mathrm{H} \alpha$ \\
\hline 6695 & 17 & 6.1 & He I 6678 \\
\hline 7093 & 34 & 10 & He I 7065 \\
\hline 7240 & 26 & 7.6 & O I 7254 \\
\hline 7465 & 6.6 & 1.8 & O I 7477 \\
\hline 7776 & 36 & 8.8 & O I 7772,7774 \\
\hline
\end{tabular}

Notes. $F(\lambda)$ : measured fluxes. $I(\lambda)$ : intensities corrected for $E(B-V)=$ 0.92 .

The contributions from He I 5015 and Fe II 5018 were very small in the emission band at $5015 \AA$. The intensity ratio $I(\mathrm{He} \mathrm{I} 5015) / I(\mathrm{He} \mathrm{I} 6678)$ is 0.778 for the Case B at $T_{\mathrm{e}}=10^{4} \mathrm{~K}$ and $N_{\mathrm{e}}=10^{7} \mathrm{~cm}^{-3}$ (Brocklehurst 1972), and this ratio is a slow function of $T_{\mathrm{e}}$ and $N_{\mathrm{e}}$. Therefore, the emission line He I 5015 occupied only $7 \%$ of the emission band at $5015 \AA$ on 2000 May 20 (Table 3). The contribution from Fe II 5018 was also small, because the emission line Fe II 4924, whose intensity is comparable to that of Fe II 5018 (Moore 1959), was very weak.

\subsection{June 9 and 10: nebular stage}

Figure 4 shows a tracing of a high-resolution spectrum obtained on 2000 June 10. All N II emission lines faded, and the [O III] 5007 emission line had replaced the N II lines at $5015 \AA$. The other forbidden lines detected in this spectrum were [O III] 4959, and [N II] 5755. Another high-resolution spectrum obtained on 2000 June 9 contained the same forbidden lines. The low-resolution spectra obtained by Kiss et al. (2001) showed no trace of [O III] line until May 30. Accordingly, this object entered the nebular stage between 2000 May 31 and June 8, that is about one month after light maximum. As reported in the next sub-section, the emission line of [O III] 5007 was prominent on 2000 July 9. On the other hand, Burlak \& Esipov (2001) reported that [O III] 5007 line had virtually disappeared on 2000 July 24. Therefore, the duration of the nebular stage was only one month and a few weeks.

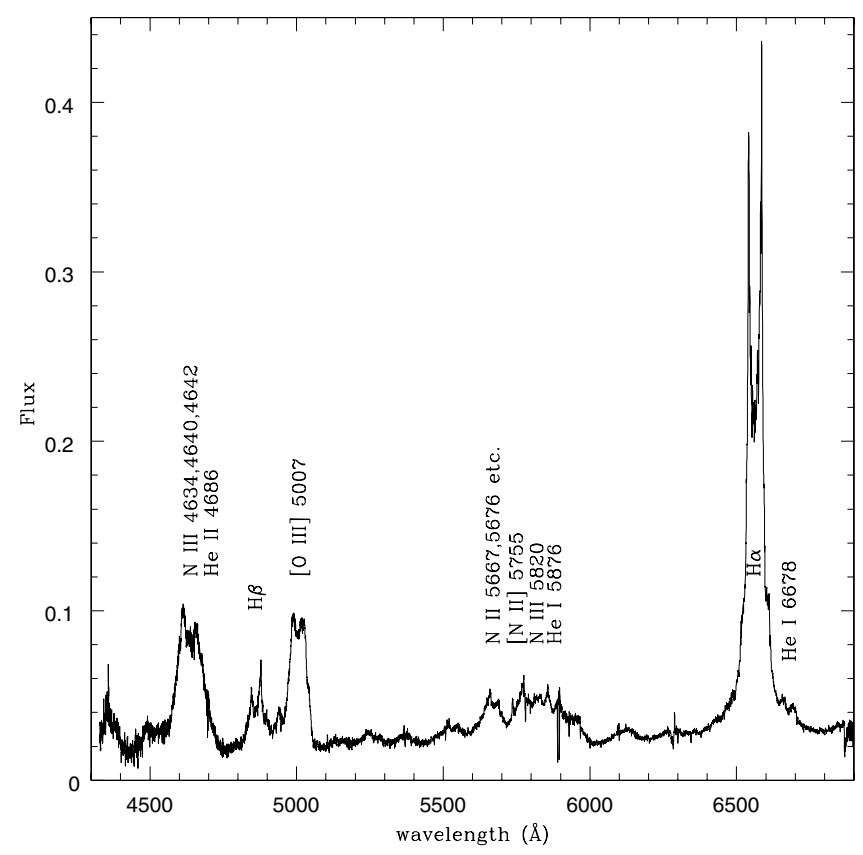

Fig. 4. High-resolution spectrum of CI Aql on 2000 June 10. The ordinate shows the flux in $10^{-12} \mathrm{erg} \mathrm{cm}^{-2} \mathrm{~s}^{-1} \AA^{-1}$.

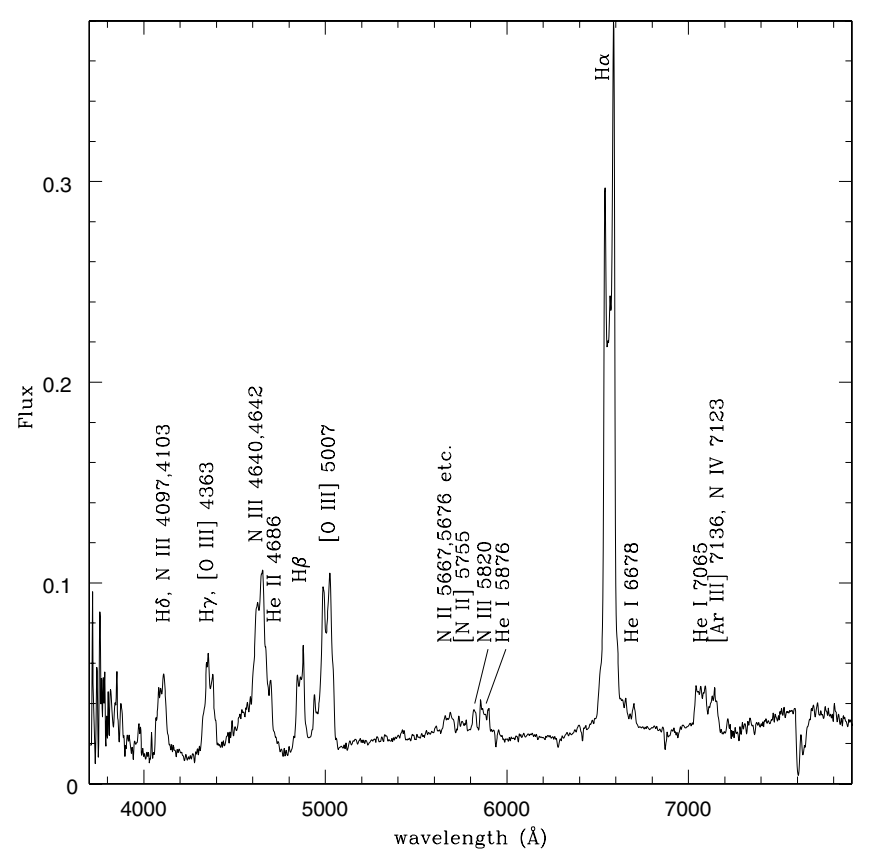

Fig. 5. Low-resolution spectrum of CI Aql on 2000 July 9. The ordinate shows the flux in $10^{-12} \mathrm{erg} \mathrm{cm}^{-2} \mathrm{~s}^{-1} \AA^{-1}$.

\subsection{July 9: nebular stage}

Figure 5 shows a tracing of a low-resolution spectrum obtained on 2000 July 9. The emission lines of [O III] 5007, 4959, and 4363 were prominent and that of [N II] 5755 was detected. The emission lines of N III and He II strengthened, while those of $\mathrm{N}$ II weakened and the O I lines had disappeared.

The emission line at $5820 \AA$ was not detected in the spectra of any classical or recurrent novae. For example, the recurrent nova RS Oph showed numerous emission lines of various ionisation levels from H I to [Fe XIV] in the outburst in 2006, but this line was not detected (Iijima 2009). Thackeray (1977) found 
Table 4. Intensities of selected emission lines relative to $\mathrm{H} \beta=100$ on 2000 July 9.

\begin{tabular}{lccc}
\hline \hline$\lambda_{\text {obs }}$ & $F(\lambda)$ & $I(\lambda)$ & Ident. \\
\hline 3969 & 25 & 50 & H $\epsilon$, [Ne III] 3967 \\
4100 & 104 & 187 & H $\delta$, III 4104, 4097 \\
4359 & 125 & 193 & H $\gamma$, [O III] 4363 \\
4475 & 9 & 12.5 & He I 4471 \\
4538 & $29:$ & $38:$ & N III 4518, 4524, 4535, etc. \\
4640 & 244 & 293 & N III 4634, 4642, N II 4643 \\
4692 & $24:$ & $28:$ & He II 4686 \\
4863 & 100 & 100 & H $\beta$ \\
5005 & 270 & 243 & [O III] 5007, 4959 \\
5323 & 5 & 4 & Fe II 5317, 5326 \\
5420 & 9 & 6 & He II 5412, Fe II 5425 \\
5680 & 28 & 16 & N II 5676, 5680 \\
5752 & 16 & 8.8 & [N II] 5755 \\
5820 & 15 & 8.1 & N III 5818, 5821 \\
5876 & 32 & 17 & He I 5876 \\
6567 & 860 & 322 & H $\alpha$ \\
6677 & 20 & 7.2 & He I 6678 \\
7068 & 65 & 20 & He I 7065 \\
7140 & 43 & 13 & [Ar III] 7136, N IV 7123, 7127 \\
7220 & 9 & 2.6 & N II 7217? \\
\hline $\mathrm{H} \beta$ & 1.95 & 36.8 & absolute int. 10 $0^{-12} \mathrm{erg} \mathrm{cm}^{-2} \mathrm{~s}^{-1}$ \\
\hline
\end{tabular}

Notes. $F(\lambda)$ : measured fluxes. $I(\lambda)$ : intensities corrected for $E(B-V)=$ 0.92 .

an emission line at $5820.8 \AA$ in the spectra of the symbiotic nova RR Tel and identified it to have been [Ni IV] 5820.1. This identification is unlikely in the present case, because no other line of [Ni IV] was detected in our spectra. It could be identified as a blend of N III 5817.8 and 5820.6 given in the NIST database ${ }^{3}$.

The profile of [ArIII] 7135.8 emission line was different from those of the other forbidden lines (Fig. 5). Probably, there were some contributions from the lines of N IV, 7109.5, 7123.1, and 7127.2 (Moore 1959) in this emission line, because emission lines of nitrogen ions were unusually intense in the spectra of this object. Intensities of selected emission lines relative to $\mathrm{H} \beta$ are given in Table 4 . The notations are the same as in Table 3. The absolute intensity of the $\mathrm{H} \beta$ emission line was also measured, and the result is given in the bottom line of Table 4 in units of $10^{-12} \mathrm{erg} \mathrm{cm}^{-2} \mathrm{~s}^{-1}$.

\section{Variations of emission and absorption lines}

Some parameters of emission and absorption components of selected lines are summarised in Table 5. Those are the full widths at half maximum (FWHM) and the radial velocities of the line centre at half maximum (l.c.) of the emission components, the separations between two emission peaks (p.s.) and the radial velocities at the middle of two peaks (p.m.), and the radial velocities of the absorption components (abs1-4). The observational errors in the radial velocities of broad emission and absorption components are about $\pm 10 \mathrm{~km} \mathrm{~s}^{-1}$ for the high-resolution spectra (May 14, 15, and 16, and June 9 and 10), and $\pm 30 \mathrm{~km} \mathrm{~s}^{-1}$ for the low-resolution spectra (May 20 and July 9). Values with larger errors are denoted by a colon. The observational errors in the radial velocities of two narrow absorption components of Fe II 5169 at about $-2200 \mathrm{~km} \mathrm{~s}^{-1}$ (abs2) and $-1400 \mathrm{~km} \mathrm{~s}^{-1}$ (abs4) are about $\pm 7 \mathrm{~km} \mathrm{~s}^{-1}$, because they were well defined (see, Fig. 8 in Sect. 5.2).

\footnotetext{
${ }^{3}$ http://physics.nist.gov/PhyRefData/ASD/lines-form. html
}

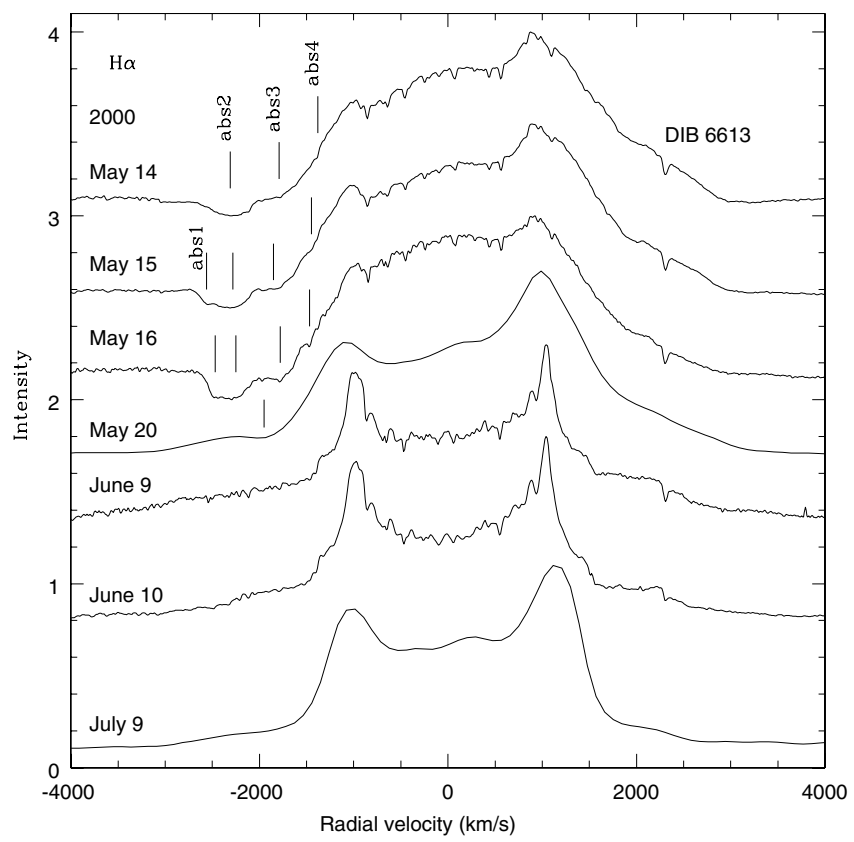

Fig. 6. Profiles of $\mathrm{H} \alpha$. The ordinate is an arbitrary intensity scale and the height of each tracing is normalised at unity. The vertical bars indicate the positions of the absorption components.

\subsection{Profiles of $\mathrm{HI}$ lines}

Figure 6 shows the profiles of $\mathrm{H} \alpha$. The ordinate is an arbitrary intensity scale and the height of each tracing is normalised at unity. The abscissa is the heliocentric radial velocity in $\mathrm{km} \mathrm{s}^{-1}$. The diffuse interstellar absorption band at $6613 \AA$ is clearly visible, and the other narrow absorption lines on $\mathrm{H} \alpha$ are atmospheric $\mathrm{H}_{2} \mathrm{O}$ bands.

Kiss et al. (2001) reported that the emission components of $\mathrm{H} \alpha$ had displayed broad Gaussian profiles in the earliest stage until 2000 May 8. The profiles of $\mathrm{H} \alpha$ on May 14-16 (Fig. 6) seem to have consisted of the broad Gaussian component and blue- and red-shifted weak emission peaks. These profiles are similar to that observed by Kiss et al. (2001) on May 15.

The vertical bars in Fig. 6 indicate the positions of the absorption components. The main absorption component (abs2) was blue-shifted by about $-2300 \mathrm{~km} \mathrm{~s}^{-1}$. Another absorption component (abs3) blue-shifted by about $-1800 \mathrm{~km} \mathrm{~s}^{-1}$ grew in depth with time during May 2000. It may be hard to notice in the tracings, but there were two additional weak absorption components: one had a higher blue-shift of about $-2500 \mathrm{~km} \mathrm{~s}^{-1}$ (abs1) and another one with lower blue-shift of about $-1400 \mathrm{~km} \mathrm{~s}^{-1}$ (abs4). The radial velocities of the absorption components of HIlines measured in the low-resolution spectrum on May 20 are denoted by a dagger in Table 5. They are probably the blends of abs2 and abs3.

Kiss et al. (2001) reported that the $\mathrm{H} \alpha$ had displayed a nearly flat-topped profile on May 25 and a clear double peak profile had first appeared on June 6. In contrast, a double peak profile was detected on May 20 (Fig. 6). The double peak profile, therefore, appeared once on May 20 and disappeared on May 25, then reappeared later. A small re-brightening is noticed around May 25 in the light curves presented by Kiss et al. (2001) and Kato et al. (2002). The temporary disappearance of the double peak profile may have been related to this re-brightening.

The line centre at half maximum of intensity (1.c.) of the emission component of $\mathrm{H} \alpha$ was red-shifted by about 
Table 5. Physical parameters of emission and absorption components of selected lines in units of $\mathrm{km} \mathrm{s}^{-1}$.

\begin{tabular}{|c|c|c|c|c|c|c|c|}
\hline 2000 & May 14 & May 15 & May 16 & May 20 & June 9 & June 10 & July 9 \\
\hline JD 245 & 1678.55 & 1679.55 & 1680.55 & 1685.25 & 1704.51 & & \\
\hline \multicolumn{8}{|c|}{$\mathrm{H} \alpha$} \\
\hline$F W H M$ & 3160 & 3190 & 3060 & 3360 & 3150 & 2970 & 2960 \\
\hline l.c. & +276 & +230 & +340 & +180 & +31 & +24 & +80 \\
\hline p.s. & 1910 & 1960 & 1950 & 2060 & 2010 & 2010 & 2160 \\
\hline p.m. & -32 & -51 & -32 & -40 & +31 & +30 & +60 \\
\hline abs1 & & -2560 & -2470 & & & & \\
\hline abs2 & -2310 & -2280 & -2250 & \multirow{4}{*}{$-1950^{\dagger}$} & & & \\
\hline abs & & & & & & & \\
\hline abs3 & $-1790:$ & -1850 & -1780 & & & & \\
\hline abs4 & $-1380:$ & $-1450:$ & -1470 & & & & \\
\hline \multicolumn{8}{|c|}{$\mathrm{H} \beta$} \\
\hline$F W H M$ & 3250 & 3150 & 2250 & 3390 & & 3300 & 3020 \\
\hline 1.c. & +318 & +380 & +400 & +210 & & +46 & +150 \\
\hline p.s. & & 2120 & & 2140 & 2000 & 1980 & 1920 \\
\hline p.m. & & +63 & & +40 & +37 & +60 & +120 \\
\hline abs1 & & -2530 & -2440 & & & & \\
\hline abs2 & -2270 & -2260 & -2230 & \multirow{4}{*}{$-1920^{\dagger}$} & & & \\
\hline abs & & & & & & & \\
\hline abs3 & -1710 & -1730 & -1730 & & & & \\
\hline abs4 & -1410 & -1430 & -1440 & & & & \\
\hline \multicolumn{8}{|c|}{ He I 5876} \\
\hline$F W H M$ & & & & & & & 2970 \\
\hline & & & & & & & +70 \\
\hline p.s. & & & & & & 1940 & 2160 \\
\hline p.m. & & & & & & -9 & +60 \\
\hline \multirow{2}{*}{\multicolumn{8}{|c|}{ Fe II 5169}} \\
\hline & & & & & & & \\
\hline abs2 & -2209 & -2211 & -2221 & & & & \\
\hline abs3 & $-1750:$ & -1733 & -1724 & & & & \\
\hline abs4 & -1398 & -1414 & -1431 & & & & \\
\hline \multicolumn{8}{|c|}{ [O III] 5007} \\
\hline p.s. & & & & & 2550 & 2180 & 2110 \\
\hline p.m. & & & & & -49 & +23 & +45 \\
\hline \multicolumn{8}{|c|}{ [N II] 5755} \\
\hline FWHM & & & & & 2360 & & 2640 \\
\hline & & & & & +58 & & +25 \\
\hline p.s. & & & & & 1980 & 1970 & 2110 \\
\hline p.m. & & & & & +38 & +40 & +90 \\
\hline
\end{tabular}

Notes. FWHM: full width at half maximum of intensity. 1.c.: line centre at half maximum of intensity. p.s.: separation of emission peaks. p.m.: middle of emission peaks. abs1-4: radial velocities of absorption components. ${ }^{(\dagger)}$ The absorption components of the H I lines in the low-resolution spectrum on May 20, whose radial velocities are denoted by a dagger, are likely the blends of abs 2 and abs 3 in the high-resolution spectra. The observational errors in the radial velocities of broad emission and absorption components are about $\pm 10 \mathrm{~km} \mathrm{~s}^{-1}$ for the high-resolution spectra (May 14, 15, and 16, and June 9 and 10), and $\pm 30 \mathrm{~km} \mathrm{~s}^{-1}$ for the low-resolution spectra (May 20 and July 9). Values with larger errors are denoted by a colon.

$+300 \mathrm{~km} \mathrm{~s}^{-1}$ on May 14, 15, and 16 (Table 5). These redshifts had disappeared in June and July. Probably, there was a very broad absorption component blue-shifted by roughly $-1000 \mathrm{~km} \mathrm{~s}^{-1}$ in the early decline stage, which cut the blue-ward side of the emission component.

The profiles of $\mathrm{H} \alpha$ on June 9 and 10 were similar to that observed by Kiss et al. (2001) on June 6. The separation of the two peaks (p.s.) was nearly constant during our observations.

The $\mathrm{H} \beta$ profiles are shown in Fig. 7. The vertical bars indicate the positions of the absorption components. A flattopped profile was seen on May 14. The profile of $\mathrm{H} \alpha$ was nearly stable during May 14 and 16 (Fig. 6), while the blueward part of the emission component of $\mathrm{H} \beta$ progressively weakened on May 15 and 16 (Fig. 7). The main absorption component of $\mathrm{H} \beta$ was blue-shifted by about $-2300 \mathrm{~km} \mathrm{~s}^{-1}$ as in $\mathrm{H} \alpha$. The other absorption components with radial velocities of $-2500 \mathrm{~km} \mathrm{~s}^{-1},-1700 \mathrm{~km} \mathrm{~s}^{-1}$, and $-1400 \mathrm{~km} \mathrm{~s}^{-1}$, were more clearly seen compared to those of $\mathrm{H} \alpha$. Red-shifts of the line centre by about $+300 \mathrm{~km} \mathrm{~s}^{-1}$ were observed in $\mathrm{H} \beta$ as well. The profiles of $\mathrm{H} \alpha$ and $\mathrm{H} \beta$ resembled each other on June 10 and July 9.

\subsection{Absorption components of Fe II 5169}

Figure 8 shows the spectral region from $5100 \AA$ to $5200 \AA$ of the high-resolution spectrum obtained on May 15. Three absorption lines are seen at 5130.9, 5139.2, and 5144.6 $\AA$. The absorption line at $5139.2 \AA$ (abs3) seems to consist of two or more components, because it is broader than the others. Kiss et al. (2001) found similar absorption lines in the spectrum obtained at May 4.4 UT and identified them as the absorption components of Fe II 5169. Their radial velocities were $-2200 \mathrm{~km} \mathrm{~s}^{-1}$, $-1700 \mathrm{~km} \mathrm{~s}^{-1}$, and $-1100 \mathrm{~km} \mathrm{~s}^{-1}$ (Kiss et al. 2001). We were able to re-analyse the spectrum at May 4.4 UT in our observatory with our method by courtesy of Dr. L.L. Kiss and slightly 
T. Iijima: Spectroscopic observations of CI Aql

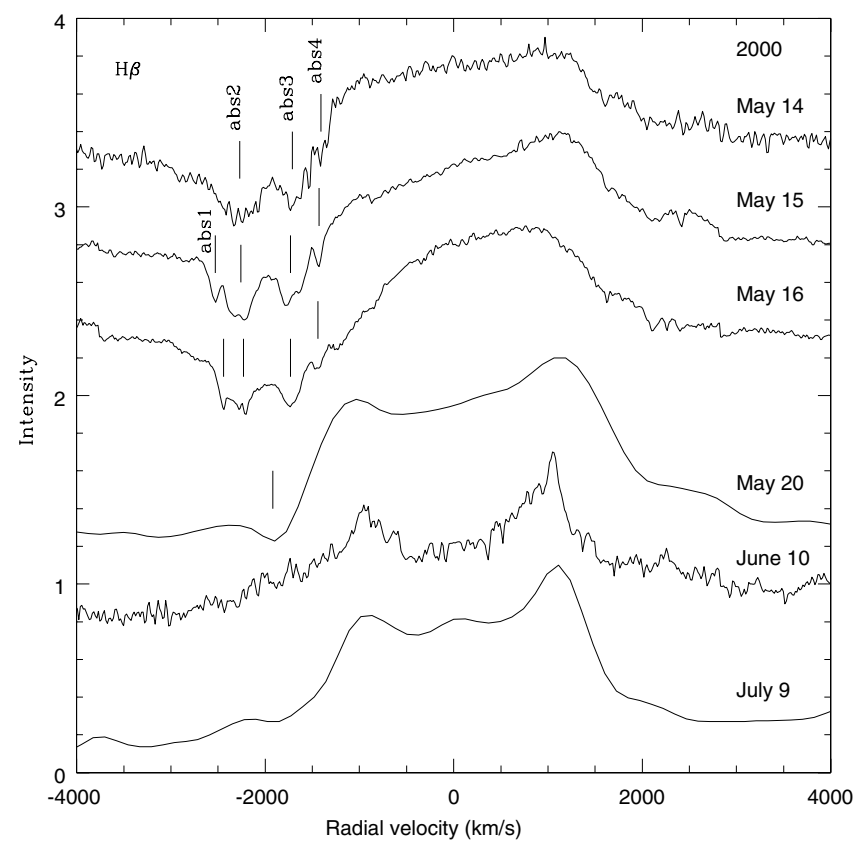

Fig. 7. Profiles of $\mathrm{H} \beta$. The ordinate is an arbitrary intensity scale and the height of each tracing is normalised at unity. The vertical bars indicate the positions of the absorption components.

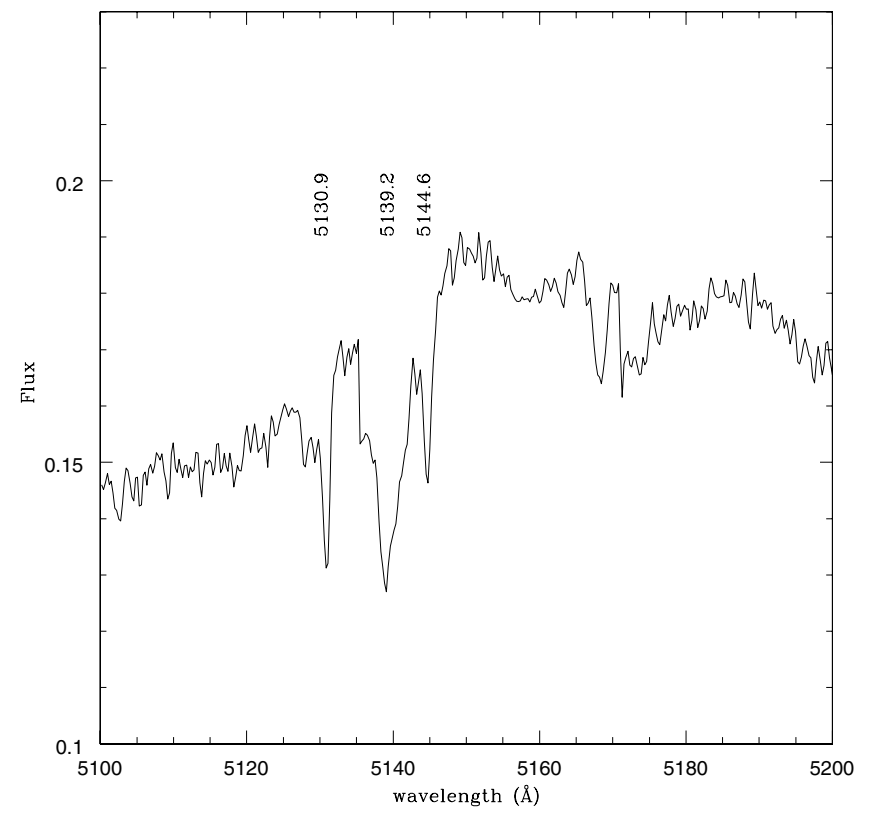

Fig. 8. Three absorption components of Fe II 5169 on 2000 May 15. The ordinate shows the flux in $10^{-12} \mathrm{erg} \mathrm{cm}^{-2} \mathrm{~s}^{-1} \AA^{-1}$.

different results were obtained: i.e. $-2190 \mathrm{~km} \mathrm{~s}^{-1},-1700 \mathrm{~km} \mathrm{~s}^{-1}$, and $-1150 \mathrm{~km} \mathrm{~s}^{-1}$ with the errors of about $\pm 20 \mathrm{~km} \mathrm{~s}^{-1}$. These results are used as those at May 4.4 UT in this paper.

The radial velocities of the absorption components of Fe II 5169 are plotted in Fig. 9. The observational errors are comparable to the sizes of the symbols. The first two components of higher blue-shifts did not change significantly. On the other hand, the last component exhibited an obvious variation in its radial velocity that is well fitted by

$v=3187.5 t^{-1 / 3}-2539.6 \mathrm{~km} \mathrm{~s}^{-1}$,

where $t=\mathrm{JD}-2451656.8$. The fitted curve is drawn with a solid line in the figure.

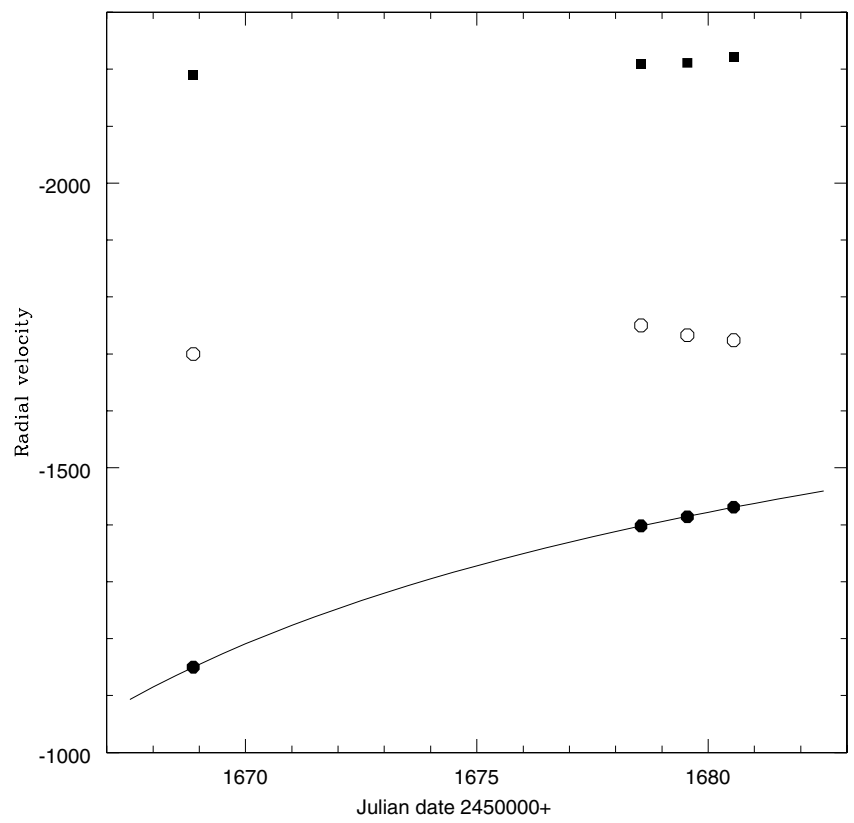

Fig. 9. Radial velocities of the absorption components of Fe II 5169 in $\mathrm{km} \mathrm{s}^{-1}$. First three points at JD 2451668.9 are measured on the spectrum obtained by Kiss et al. (2001). The best-fitted curve for the lowest velocity component, given by Eq. (1), is drawn with a solid line.

The last absorption component was probably ejected later than the others, because it was still accelerating during the early decline stage. Extrapolating Eq. (1), we have $v=0$ at JD2451659, namely 2000 April 24. Probably, the last component was ejected around this date. The first two components were likely ejected at a certain epoch or epochs earlier than April 24. These results suggest that the major outburst of CI Aql in 2000 occurred earlier than the date of the discovery of the outburst on April 28. The first two components were likely accelerated by a different mechanism because very long times are required to reach their velocities with the acceleration given by Eq. (1), i.e. two months for $-1700 \mathrm{~km} \mathrm{~s}^{-1}$ and 2 years for $-2200 \mathrm{~km} \mathrm{~s}^{-1}$.

It seems that the absorption components of the lowest radial velocities (abs4) of $\mathrm{H} \alpha$ and $\mathrm{H} \beta$ were also accelerating during our observations (Table 5). Detailed studies of these phenomena, however, are difficult to perform because of the lack of data in the earlier epoch.

The rest velocity of CI Aql system is estimated to be $+35 \pm$ $2 \mathrm{~km} \mathrm{~s}^{-1}$ by measuring the radial velocities of the line centres of some isolated emission lines at the nebular stage.

\section{Properties of ejecta}

\subsection{Electron density}

The electron density in the ejected gas shells is estimated from the intensity ratio of the nebular lines to the auroral line of [O III]. The emission lines of $\mathrm{H} \gamma$ and [O III] 4363 were merged in the low resolution spectrum (Fig. 5). Therefore, the intensity of [O III] 4363 line is estimated assuming the theoretical intensity ratio of $\mathrm{H} \gamma$ to $\mathrm{H} \beta$. A very high electron density was expected in the ejected gas shells at that time, because the intensity of [O III] 4363 emission line was roughly of the same order as that of [O III] 5007 (Fig. 5). The intensity ratio of $\mathrm{H} \gamma$ to $\mathrm{H} \beta$ under such a high density condition is $I(\mathrm{H} \gamma) / I(\mathrm{H} \beta)=0.49$ (Hummer \& Storey 1987). The intensity of the [O III] 4363 relative to $I(\mathrm{H} \beta)=100$ is estimated to be 144 from the intensity of 
the blend of $\mathrm{H} \gamma$ and [O III] 4363 given in Table 4. The intensity ratio of the nebular lines to the auroral line of [O III] is

$[I(5007)+I(4959)] / I(4363)=1.69$.

Using the formula of Seaton (1975), the electron density in the ejecta is estimated to be $5.0 \times 10^{7} \mathrm{~cm}^{-3}$. The adopted electron temperature is $11000 \mathrm{~K}$, which is the mean electron temperature in the ejecta of classical novae at nebular stage given by Snijders (1990).

\subsection{Helium abundance and mass of ejecta}

The helium abundance in the ejecta is derived from the intensities of He I 4471, He I 5876, He I 6678, and He II 4686 emission lines on 2000 July 9 (Table 4). The He I 7065 is not used in the estimate, because it was blended with [Ar III] 7135, and N IV 7109 and 7123 (Sect. 4.4). The intensity of He II 4686 was uncertain, because it was blended with the much stronger emission line at $4640 \AA$ (Fig. 5). Fortunately, the error in the helium abundance owing to the ambiguity in the intensity of He II 4686 is small, because the fraction of ionised helium was low at that period. We derive $N(\mathrm{He})=0.19 \pm 0.05$, where $N\left(\mathrm{He}^{0}\right)=0.16$ and $N\left(\mathrm{He}^{+}\right)=0.03$ from the formulae given by Iijima (2006). The effects of the collisional excitation of the He I lines are corrected using the formulae of Peimbert \& Torres-Peimbert (1987). This result agrees with the value $N(\mathrm{He})=0.22$ derived by Burlak \& Esipov (2001).

The mass of the ejecta is estimated to be about $2 \times 10^{-6} M_{\odot}$ from the intensities of H I, He I, and He II lines using the formulae given by Iijima (2006), where the adopted distance is $1.55 \mathrm{kpc}$. This result should be a lower limit for the mass of the ejecta, because only the gas related to the emission lines is taken into account. This result is consistent with the mass loss $2.2 \times 10^{-6}<\Delta M<5.7 \times 10^{-6} M_{\odot}$ derived by Lederle $\&$ Kimeswenger (2003) from the change of the orbital period through the outburst in 2000.

\section{Doubts about light maximum of the outburst in 2000}

One low-resolution spectrum was obtained at 2000 April 29.6 UT by Uemura \& Kato (2000). They inferred that the object was in the post-maximum stage, because the spectrum contained prominent H I emission lines without any P Cygni type absorption component. Matsumoto et al. (2001) carried out a detailed analysis for the same spectrum and found out the Fe II, He I, He II, N II, and N III emission lines, in addition to H I lines. This result supports the interpretation of Uemura \& Kato (2000), because these lines, except for H I and Fe II, do not appear in the spectra of classical novae at the pre-maximum stage (McLaughlin 1960). The luminosity of the object, however, increased after their observation and reached the peak luminosity of $m_{\mathrm{V}} \cong 9 \mathrm{mag}$ at 2000 May 5.0 UT (Kiss et al. 2001).

Two interpretations are likely for the behaviour of this object. One is that CI Aql is an extremely peculiar object which showed the post-maximum spectrum at the pre-maximum stage. The other one is that CI Aql was indeed at the post-maximum stage at 2000 April 29.6 UT, i.e. the main outburst occurred earlier than April 29.6, which had been overlooked in the patrol of nova watchers. This hypothesis is not too arbitrary, because there was a full moon on 2000 April 18. The spectral data support this hypothesis, because the gas shells were probably ejected around 2000 April 24 or still earlier (Sect. 5.2).
There is also a doubt about the maximum luminosity in the outburst in 2000. Matsumoto et al. (2001) called attention to the work of Williams (2000), who estimated the maximum luminosity of the outburst in 1917 to have been $8.6 \mathrm{mag}$ in the Tycho $\mathrm{B}$ band according the survey of Harvard College Observatory Patrol plates. The difference between the Tycho $B$ band and the Johnson $B$ band is not important in this argument. Therefore, it is written simply as $B$ in the successive part of this article. The intrinsic colours of classical novae at maximum luminosity are found to be in the range $(B-V)_{0}=0.23 \pm 0.16$ (Warner 1995). The maximum luminosity of the outburst in 1917 was, therefore, probably $m_{\mathrm{V}}=7.6 \mathrm{mag}$ or brighter, because CI Aql is highly reddened by $E(B-V)=0.92$. The observed maximum luminosity, $m_{\mathrm{V}} \cong 9$ mag (Kiss et al. 2001), in the outburst in 2000 was much fainter than that in 1917.

If we adopt $m_{\mathrm{V}}=7.6 \mathrm{mag}$ as the maximum luminosity of the outburst in 2000 , the absolute maximum luminosity $M_{\mathrm{V}}=$ -7.4 mag estimated by Burlak \& Esipov (2001) gives the distance $2.7 \mathrm{kpc}$, whereas we derive $2.6 \mathrm{kpc}$ with the result of Kiss et al. (2001). These distances still exceed the $1.55 \mathrm{kpc}$ derived in the theoretical model (Hachisu et al. 2003), but the disagreements become small. If the luminosity of CI Aql in the outburst in 2000 reached $m_{\mathrm{V}}=7.0 \mathrm{mag}$, we derive a distance $2 \mathrm{kpc}$.

The spectroscopic and photometric data suggest that there was the true light maximum of $m_{\mathrm{V}}=7.6 \mathrm{mag}$ or brighter at a time earlier than the discovery of the outburst on 2000 April 28.

\section{Classification of $\mathrm{Cl} \mathrm{Aql}$}

The recurrent novae have been classified into three groups according to the orbital periods of their binary systems (Warner 1995). The T Pyx type (T Pyx and IM Nor) have $P_{\text {orb }} \cong 0.1$ day, U Sco type (U Sco, V394 CrA, and Nova LMC 1990-2) have $P_{\text {orb }} \cong 1$ day, and T CrB type (T CrB, RS Oph, V4745 Sco, etc.) have $P_{\text {orb }}>100$ days. The properties of IM Nor, which was not recognised as a recurrent nova when the review by Warner (1995) was published, are given by Kato et al. (2002) and Woudt $\&$ Warner (2003). Schaefer (2010) recently proposed to divide the recurrent novae into two groups: i.e. T Pyx type (T Pyx and IM Nor) and the others. This classification seems to be too simple, because the large differences between $\mathrm{T}$ CrB type and the others are ignored.

CI Aql has been classified as a U Sco type (Kiss et al. 2001; Lynch et al. 2004), because its orbital period is 0.62 day (Mennickent \& Honeycutt 1995). The spectral evolution of CI Aql during the outburst in 2000, however, resembled those of T Pyx type recurrent novae. The spectral evolution of T Pyx in the outburst in 1966 was reported by Catchpole (1969), and that of IM Nor in 2002 was studied by Duerbeck et al. (2002) and Liller (2002). The spectra of CI Aql at the early decline stage (Figs. 2 and 3) resemble that of T Pyx in the outburst in 1966 at day 92.3 , denoted as D7563 by Catchpole (1969), and the spectrum on July 9 (Fig. 5) is similar to that at day 142.3 (D7629).

The three objects T Pyx, IM Nor, and CI Aql showed the prominent [O III] and [NII] lines in the outbursts: T Pyx (Catchpole 1969), IM Nor (Duerbeck et al. 2002; Liller 2002), and CI Aql (Burlak \& Esipov 2001; this paper). In contrast, no forbidden line was detected in the outbursts of U Sco type recurrent novae until Diaz et al. (2010) found the lines of [O III], [N II], and [Ne III] in the last outburst of U Sco in 2010: U Sco (Rosino \& Iijima 1988; Sekiguchi et al. 1988; Munari et al. 1999; Iijima 2002), V394 CrA (Sekiguchi et al. 1989), and Nova LMC 1990-2 (Sekiguchi et al. 1990). 
The spectral evolution of U Sco was different from those of T Pyx, IM Nor, and CI Aql even though the forbidden lines were detected. The broad emission components of H I lines nearly disappeared at day 23 in the outburst of U Sco in 2010 (Diaz et al. 2010), then the forbidden lines were first detected at day 51. In contrast, in the cases of T Pyx, IM Nor, and CI Aql, the [O III] and $[\mathrm{N} \mathrm{II}]$ emission lines appeared when the H I emission lines were still broad. Therefore, the forbidden lines appeared in the spectrum of U Sco at a much later stage of its evolution with respect to those of T Pyx, IM Nor, and CI Aql.

Kato et al. (2002) and Duerbeck et al. (2002) pointed out that the light curves of T Pyx, IM Nor, and CI Aql resembled those of medium-fast classical novae. Their light curves, therefore, are different from those of U Sco (Rosino \& Iijima 1988; Sekiguchi et al. 1988), V394 CrA (Sekiguchi et al. 1989), and Nova LMC 1990-2 (Sekiguchi et al. 1990), which were characterised by the extremely fast fading.

The spectroscopic and photometric properties of CI Aql are similar to those of T Pyx type recurrent novae, even though the orbital period is close to those of U Sco type. It seems that T Pyx, IM Nor, and CI Aql form a sub-group of recurrent novae. This classification has been suggested also in some previous works (Anupama 2002; Duerbeck et al. 2002; Kato et al. 2002).

\section{Concluding remarks}

The optical spectra of the recurrent nova CI Aql during the outburst in 2000 are presented. The amount of interstellar extinction is estimated to be $E(B-V)=0.92 \pm 0.15$ from the equivalent widths of the diffuse interstellar absorption bands (DIB). Using the intensities of selected emission lines at the nebular stage, the helium abundance is estimated to be $N(\mathrm{He})=0.19 \pm 0.05$, which agrees with the result of Burlak \& Esipov (2001). The mass of the ejecta on 2000 July 9 is estimated to be about $2 \times 10^{-6} M_{\odot}$.

Three or four absorption components were detected on the $\mathrm{HI}$ and Fe II lines, among which that of Fe II with the lowest blue-shift was still accelerating during the early decline stage. The ejections of gas shells may have occurred earlier than the date of the discovery of the outburst on 2000 April 28.

The early appearance of the emission lines of [O III] and [NII] suggests a possible classification of CI Aql as a T Pyx type recurrent nova. However, further works are needed for the classification, because CI Aql and T Pyx are different in the orbital periods of their binary systems. The duration of the nebular stage was only one month and a few weeks. The free expansion of the ejected gas shells into the interstellar space did probably not occur, because the electron density in the ejected shells was high, $5.0 \times 10^{7} \mathrm{~cm}^{-3}$, even at the nebular stage. The disappearance of the emission lines of [O III] 5007 and 4959 at the end of July 2000 was probably caused by a rising in electron density in the ejecta. It is unlikely that significant compression of the ejecta occurred by a collision with an outer circumstellar envelope. It seems that the ejected gas shell started to shrink at that time. If this is the case, we have to take into account the effect of the recovering matter in the evolutionary scenario of this system.

As mentioned in Sect. 7, there are doubts about the epoch and magnitude of light maximum of the outburst in 2000 . We have to wait for the next outburst to resolve the problems presented in this paper.

Acknowledgements. I am grateful to $\mathrm{R}$. Barbon for the careful reading of the manuscript and useful suggestions. Thanks are also due to T. Matsuda, I. Hachisu, and M. Kato for helpful discussions. I am indebted to K. Matsumoto, K. Ayani, and T. Kawabata for assistance to use the spectra of CI Aql in the archives of the Bisei Astronomical Observatory, and to L. L. Kiss to use the spectrum at 2000 May 4.4 UT.

\section{References}

Anupama, G. C. 2002, in Classical Nova Explosions, eds. M. Hernany, \& J. José, AIP Conf. Proc., 637, 32

Brocklehurst, M. 1972, MNRAS, 157, 211

Burlak, M. A., \& Esipov, V. F. 2001, Astron. Lett., 27, 674

Catchpole, R. M. 1969, MNRAS, 142, 119

Diaz, M. R., Williams, R. E., Luna, G. J., Moraes, M., \& Takeda, L. 2010, AJ, 140,1860

Duerbeck, H. W., Sterken, C., Baptista, R., et al. 2002, in Classical Nova Explosions, eds. M. Hernany, \& J. José, AIP Conf. Proc., 637, 299

Hachisu, I., \& Kato, M. 2001, ApJ, 553, L161

Hachisu, I., Kato, M., \& Schaefer, B. E. 2003, ApJ, 584, 1008

Hummer, D. G., \& Storey, P. J. 1987, MNRAS, 224, 801

Iijima, T. 2002, A\&A, 387, 1013

Iijima, T. 2006, A\&A, 451, 563

Iijima, T. 2009, A\&A, 505, 287

Jenniskens, P., \& Désert, F.-X. 1994, A\&AS, 106, 39

Kato, T., Yamaoka, H., Liller, W., \& Monardo, B. 2002, A\&A, 391, L7

Kerr, F. J., \& Westerhout, G. 1965, in Galactic Structure, eds. A. Blaauw, \& M. Schmidt (The University of Chicago Press), 167

Kiss, L. L., Thomson, J. R., Oglaza, W., Fürész, G., \& Sziládi, K. 2001, A\&A, 366,858

Lederle, C., \& Kimeswenger, S. 2003, A\&A, 397, 951

Liller, W. 2002, in Classical Nova Explosions, eds. M. Hernany, \& J. José, AIP Conf. Proc., 637, 289

Lynch, D. K., Wilson, J. C., Rudy, R. J., et al. 2004, AJ, 127, 1089

McLaughlin, D. B. 1960, in Stellar Atmospheres, ed. J. L. Greenstein (The University of Chicago Press), 585

Matsumoto, K., Uemura, M., Kato, T., et al. 2001, A\&A, 378, 487

Mennickent, R. E., \& Honeycutt, R. K. 1995, IBVS, No. 4232

Moore, C. E. 1959, A Multiplet Table of Astrophysical Interest (Washington DC: US Dept. of Commerce, Office of Technical Services)

Munari, U., Zwitter, T., Tomov, T., et al. 1999, A\&A, 347, L39

Münch, G. 1968, in Nebulae and Interstellar Matter, eds. B. M. Middlehurst, \& L. H. Aller (The University of Chicago Press), 365

Oudmaijer, R. D., Busfield, G., \& Drew, J. E. 1997, MNRAS, 291, 797

Peimbert, M., \& Torres-Peimbert, S., 1987, Rev. Mex. Astron. Astrofis., 14, 540 Reinmuth, K. 1925, AN, 225, 385

Rosino, L., \& Iijima, T. 1988, A\&A, 201, 89

Schaefer, B. E. 1990, ApJ, 355, L39

Schaefer, B. E. 2001, IAU Circ. No. 7750

Schaefer, B. E. 2010, ApJS, 187, 275

Seaton, M. J. 1975, MNRAS, 170, 475

Sekiguchi, K., Feast, M. W., Whitelock, P. A., et al. 1988, MNRAS, 234, 281

Sekiguchi, K., Catchpole, R. M., Fairall, A. P., et al. 1989, MNRAS, 236, 611

Sekiguchi, K., Stobie, R. S., Buckley, D. A. H., \& Caldwell, J. A. R. 1990, MNRAS, 245, 28

Snijders, M. A. J. 1990, in Physics of Classical Novae, eds. A. Cassatella, \& R. Viotti (Springer-Verlag), 188

Takamizawa, K., Kato, T., Yamamoto, M., et al. 2000, IAU Circ. No. 7409

Thackeray, A. D. 1977, Mem. R. Astr. Soc., 83, 1

Uemura, M., \& Kato, T. 2000, IAU Circ. No. 7409

Warner, B. 1995, Cataclysmic Variable Stars (Cambridge Univ. Press)

Williams, D. B. 2000, IBVS, No. 4904

Woudt, P. A., \& Warner, B. 2003, MNRAS, 343, 313 